\title{
Suizidbeihilfe und Urteilsfähigkeit aus psychiatrischer Sicht
}

Mario Gmür

Korrespondenz:

PD Dr. med. Mario Gmür Rämistrasse 3

CH-8001 Zürich

mario_gmuer@bluewin.ch

\begin{abstract}
Einleitung
Die Beihilfe zum Suizid ist Neuland auch für die Psychiatrie. Wer auf diesem Gebiet heute keinen Mangel an Erfahrung aufweist, wirkt suspekt und gehört zu jenen, die Anlass zum Ruf nach einer Regelung geben, die für Seriosität und Sorgfalt bei der Durchführung von Suizidbeihilfe bürgen soll. Wo es an Erfahrung mangelt, hat die Tugend der weisen Voraussicht in die Lücke zu springen und ist man darauf angewiesen, aus Erfahrungen in ähnlichen Bereichen und bei analogen Problemstellungen zu schöpfen und die daraus abgeleiteten Konzepte mutatis mutandis auf die neuen Herausforderungen zu übertragen.
\end{abstract}

\section{Ethische Aspekte}

Der verantwortungsbewusste Psychiater, der sich über die Folgen seines Wirkens als Gutachter Gedanken macht, hat ein legitimes Interesse zu wissen, in welcher Weise die von ihm angefertigten Gutachten vom Auftraggeber verwendet werden. Wenn er die Urteilsfähigkeit von Sterbewilligen, die um Beihilfe zum Suizid bitten, abklärt, tut er dies heute in einem Klima, in dem viele brisante philosophische, ethische und politische Fragen kontrovers beurteilt werden. Er befindet sich in einem Spannungsfeld zwischen

- gebotener Fürsorge und dem Respekt vor der Selbstbestimmung eines Suizidwilligen;

- der rechtspolitisch gebotenen Aufsicht und dem Respekt vor Individualität und Intimität des Suizidwilligen;

- einerseits einer traditionellen Haltung, die dem Leben göttlichen Ursprung zuweist und die Ehrfurcht vor der Krone der Schöpfung absolut setzt, und andererseits einer modernutilitaristischen, rational-ethischen Einstellung, die das Eingreifen in natürliche Vorgänge recht bedenkenlos praktiziert;

- den Exponenten einer umfassend liberalen Praxis, die die Suizidbeihilfe für alle lebensmüden Menschen freigeben wollen, und jenen gemässigten Befürwortern der Sterbehilfe, die diese nur bei unheilbar Kranken in der terminalen Phase als zulässig betrachten;

- der Ansicht, dass ärztliche Suizidassistenz zum Pflichtenheft des Arztes gehört, und der

\section{Assistance au suicide et capacité de discernement du point de vue psychiatrique}

L'assistance au suicide est une notion nouvelle en psychiatrie aussi. Toute personne expérimentée dans ce type d'aide est forcément suspecte et fait partie de ceux qui incitent la société à exiger l'instauration d'une réglementation garantissant que I'assistance au suicide soit pratiquée avec sérieux et diligence. En revanche, si la personne manque d'expérience, sagesse et prévoyance doivent pallier ses lacunes et elle est amenée à s'inspirer de ses expériences dans des domaines apparentés mais posant des problèmes similaires pour ensuite pouvoir les appliquer, moyennant les modifications nécessaires, aux nouveaux défis qui se présentent.

Auffassung, dass sie vom Arzt nicht verlangt werden darf;

- den Minimalisten, welche die Vorabklärungen aus Rücksicht auf den Sterbewilligen auf ein Minimum beschränken wollen und einer Expressbegleitung das Wort reden, und den Befürwortern eines langwierigen, gründlichen und daher belastenden Abklärungsverfahrens, das dem Anwärter auf Suizidhilfe einen anstrengenden Hindernislauf zumutet.

Wer sich an der Sterbehilfe beteiligt, sollte sich meines Erachtens nicht den Einwänden und skeptischen Stimmen verschliessen, die von den radikalen Befürwortern oft mit despektierlichem Unterton als sogenannte «slippery slope»-Einwände abqualifiziert werden [1]

Folgende Bedenken sind $\mathrm{zu}$ notieren und ernst zu nehmen:

1. Die Gefahr eines Dammbruches, indem eine allgemeine Erwartungshaltung aufkommt, welche Menschen leicht zum assistierten Suizid hinlenkt und diesen niederschwellig anbietet. 
2. Die Entstehung einer Todes- und Suizidkultur mit Nachahmungseffekten, begünstigt durch eine allgemeine Trivialisierung des Freitodes, ja des Todes überhaupt.

3. Die verschleierte Diskriminierung von Menschen, die durch Invalidität und Pflegeaufwand zur Last fallen, u.a. durch subtile Pressionen.

4. Das stille Einschleichen einer spartanischen Selektionsphilosophie mit eliminatorischer Haltung gegenüber unwertem, sinnlosem, unerträglichem und störendem Leben, und damit verkappte selbstsüchtige Motive des engeren und weiteren Umfeldes.

5. Die Gefahr der voreiligen Entscheidungsfindung, auf welche immer schon, vor der aktuellen Diskussion über die Suizidbeihilfe, durch Zitierung katamnestischer Untersuchungen hingewiesen wurde, die, wenn auch nicht bei Menschen in terminalem Stadium einer Krankheit, zeigen, dass $80 \%$ von Personen, die einen Suizidversuch überlebt haben, die Tat nicht wiederholen würden [2].

Das uneingeschränkte, vorbehaltlose Vertrauen in die Selbstbestimmung des Sterbewilligen bedarf ebenfalls einer kritischen Betrachtung. In meinem Buch «Die Unfähigkeit zu zweifeln» [3] habe ich als eine Kernaussage die These formuliert, dass unsere Autonomie in der Bildung von Überzeugungen weniger authentisch ist, als wir uns dies gemeinhin vorstellen. Wir wiegen uns in der Illusion von Authentizität, um unser eigenes Identitätsbedürfnis zu befriedigen, sind aber bei der Bildung unserer Überzeugungen abhängig von vielen äusseren und inneren Einflüssen wie z.B. von der Symbolik, Triebhaftigkeit, Gehirnfunktionen, Gruppenkonstellationen, massenpsychologischen Vorgängen, identitätsstiftenden und Anpassungsmechanismen, Einflüssen, deren wir uns nicht oder nur minimal bewusst sind. Eine Relativierung der Autonomie bietet allerdings keine tragfähige Grundlage für die verbindliche Regelung des Zusammenlebens, das nur funktionieren kann, wenn wir bei allen Subjekten von einem Zentrum der Verantwortung ausgehen können, sozusagen einer Als-ob-Autonomie. Vor allem das Rechtsleben ist auf die Stabilität eines voll zuständigen Subjekts angewiesen, das, auch wenn es auf der sandigen Grundlage einer Als-ob-Autonomie gebaut ist, verbindlich beim Wort genommen werden kann. Für die Beurteilung der heiklen Thematik von Sterbewillen und Verlangen nach Suizidbegleitung ist aber der Brüchigkeit von Autonomie Rechnung zu tragen, wenn wir uns nicht unbekümmert von der manifesten Willensäusserung des Sterbewilligen füh- ren lassen wollen. Daher sind die Bedenken des Psychiaters R. Pomeranz ernst zu nehmen, «dass ein Gutachten ausserhalb einer therapeutischen Beziehung als Grundlage zur Befürwortung der Suizidbeihilfe bei Menschen mit psychischen Störungen notwendigen Qualitätsanforderungen nicht genügt» [1].

Die Vorstellung vom «freien Suizid» oder vom «Freitod», auch «Bilanzsuizid» genannt, die dank Jean Amerys Plädoyer für den Freitod weite Verbreitung gefunden hat, wurde von psychiatrischer Seite in Zweifel gezogen. Asmus Finzen zweifelt daran, weil jede drastisch negative Bilanz dem suizidalen Menschen von aussen durch widrige Umstände oder Misserfolge aufgezwungen sei [4]. Thomas Bronischer verneint die Möglichkeit freier Entscheidungsfähigkeit, weil Suizidversuche meist Impulshandlungen darstellten, nämlich im Affekt der Verzweiflung und Hoffnungslosigkeit erfolgten [5].

\section{Vorgespurte Wege und eingeschliffene Terminologie}

Wer heute in die Diskussion über die Sterbehilfe einsteigt, findet bereits mehr oder weniger ausgereifte Konzepte und eine eingeschliffene Terminologie vor, die er übernehmen muss, um sich verständlich zu machen und aus dem Diskurs nicht ausgeschlossen $\mathrm{zu}$ werden, die aber einer kritischen Hinterfragung bedürfen. So ist beispielsweise der Ausdruck Sterbehilfe vieldeutig und in der häufigsten Form seiner Verwendung irreführend. Er könnte bedeuten sowohl helfen beim Sterben im Sinne der Palliative Care durch Schmerzlinderung und psychischen Beistand als auch helfen $z u$ sterben im Sinne der Lebensverkürzung. Auch der Ausdruck passive Sterbehilfe erscheint bei unbefangener Prüfung als unkorrekt, denn es handelt sich beim damit gemeinten Abbruch einer Behandlung nicht um eine Beschleunigung des Sterbevorganges, sondern darum, die Verhinderung des natürlichen Sterbevorganges zu beenden.

Nicht nachvollziehbar z. B. ist auch der Standpunkt, dass Sterbehilfe grundsätzlich nicht mit dem auf Krankheitsheilung und Schmerzlinderung ausgerichteten Ziel ärztlicher Tätigkeit vereinbar sei. In den medizinisch-ethischen Richtlinien der SAMW wird diese Ansicht wie folgt formuliert: «Auf der einen Seite ist die Beihilfe zum Suizid nicht Teil der ärztlichen Tätigkeit, weil sie den Zielen der Medizin widerspricht [...].» [6] Die Medizin kann durchaus unterschiedliche und auch gegensätzliche Ziele verfolgen, wie beispielsweise Abstinenz und Substitutionsbehandlung bei Heroinabhängigen oder Psychotherapie und Begutachtung in der psychiatrischen Praxis. 
Verständlich ist, dass der einzelne Arzt eine für ihn persönlich ethisch nicht vertretbare Methode verweigert und dass die Medizin miteinander unvereinbare Behandlungen nicht unter dem gleichen Dach anbietet, um die für die ärztliche Motivation und den ungestörten Verlauf der Behandlung notwendige Identität zu sichern. Schliesslich mutet auch das ausnahmslose Beharren auf der sogenannten Tatherrschaft des Sterbewilligen über die todbringende Handlung, trotz Erfüllung aller anderen Voraussetzungen, einschliesslich der Urteilsfähigkeit, rigide und doppelbödig an, zumal gerade in solchen Fällen, wo der Suizidwillige durch seine Invalidität zur Durchführung der Selbsttötung nicht fähig ist, die Gründe für die vorzeitige Lebensbeendigung besonders überzeugend sein dürften.

\section{Die Richtlinien für die Suizidbeihilfe}

Bekanntlich versuchen Gesetzgeber sowie aufsichts- und standesrechtliche Gremien die gegensätzlichen Standpunkte zu versöhnen durch die Definition von Qualitäts- und Zulassungskriterien, an denen sich Indikation und Durchführung von Sterbehilfe orientieren sollen. Gemäss medizinisch-ethischen Richtlinien der Schweizerischen Akademie der Medizinischen Wissenschaften (SAMW) vom 25. November 2004 betreffend die Betreuung von Patientinnen und Patienten am Lebensende sind es folgende:

- die Erkrankung soll in einer terminalen Phase sein;

- alternative Möglichkeiten der Hilfestellung sollen erörtert und so weit wie erwünscht eingesetzt worden sein;

- Urteilsfähigkeit des Patienten;

- der Suizidwunsch soll wohlerwogen, ohne äusseren Druck entstanden und dauerhaft sein;

- der Suizidwunsch soll durch eine Drittperson überprüft werden;

- die Tatherrschaft des Patienten über die Suizidhandlung soll vorhanden sein.

Eine Lockerung der Indikationsbeschränkung durch das Bundesgericht im November 2006 hat vor allem bei Ärzten moralische Aufregung und Entrüstung ausgelöst. Diese betrifft die Zulassung des Bilanzsuizides bei nicht letalen, unheilbaren, dauerhaften schweren psychischen Beeinträchtigungen, die ähnlich wie eine somatische Behinderung ein Leiden begründen, das dem Patienten sein Leben auf Dauer hin nicht mehr als lebenswert erscheinen lässt [7].

\section{Die Urteilsfähigkeit}

Bei der forensisch-psychiatrischen Beurteilung der Urteilsfähigkeit von Sterbewilligen, die Bei- hilfe zum Suizid verlangen, gelten die allgemeinen Regeln und Kriterien.

Art. 16 ZGB beschreibt die Urteilsfähigkeit wie folgt: «Urteilsfähig im Sinne dieses Gesetzes ist ein jeder, dem nicht wegen seines Kindesalters oder in Folge von Geisteskrankheit, Geistesschwäche, Trunkenheit oder ähnlichen Zuständen die Fähigkeit mangelt, vernunftgemäss zu handeln.» (In der zukünftigen Gesetzesänderung wird wahrscheinlich der Begriff «Geisteskrankheit» durch den Begriff "psychische Störungen», jener der «Geistesschwäche» durch «geistige Behinderung» und jener der «Trunkenheit» durch «Rausch» ersetzt werden, wobei es sich hier mehr um eine semantische Betriebsamkeit als um eine substantiell relevante Modifikation handeln dürfte.)

Der Altmeister der schweizerischen forensischen Psychiatrie Hans Binder definierte die Urteilsfähigkeit als Fähigkeit, «die Beweggründe und Folgen seines Verhaltens richtig zu erkennen und einer richtigen Erkenntnis gemäss zu handeln» [8].

Folgende Regeln werden bei der Beurteilung der Urteilsfähigkeit beachtet:

1. Die Untersuchung soll zweiphasig erfolgen, nämlich erstens durch Überprüfung der Einsichtsfähigkeit und zweitens der Willensfähigkeit ([9]; Abs. 59, Bezugnahme auf Fussnoten 61 und 62).

2. Zu beurteilen sind nicht die Einsicht und der Wille, sondern die Fähigkeit zur Einsicht und die Fähigkeit zur Willensbildung, und zwar nur mit Bezug auf eine der im Gesetz definierten psychischen Störungen.

3. Im Gegensatz zur Schuldfähigkeit fehlt eine abgestufte Beurteilung in der rechtlichen Würdigung der Urteilsfähigkeit, es besteht eine Beschränkung auf die Alternative «urteilsfähig ja oder nein» $[10,11]$.

4. Es gilt das Prinzip der Relativität, welches besagt, dass nicht generelle Urteilsfähigkeit, sondern immer eine Urteilsfähigkeit für eine konkrete Rechtshandlung zu prüfen ist ([9]; Abs. 140).

5. Einige Rechtshandlungen wie z. B. Entgegennahme eines Geschenks, Eingriff in die körperliche Integrität bei einer Operation, Errichtung eines Testamentes werden als von höchstpersönlicher Natur eingestuft, wobei die Ansprüche an die Urteilsfähigkeit weniger hoch gestellt werden, um den Willen des Patienten so weit wie möglich zu respektieren.

6. Es gilt die Umkehrung der Beweislast, wenn die handelnde Person ihrer allgemeinen Verfassung nach im Normalfall und mit Wahrscheinlichkeit als urteilsunfähig gelten muss [12]. Bei einem schwer dementen Kranken in 
einem Pflegeheim oder einem chronischen Wahnkranken ist also die Urteilsfähigkeit zu beweisen, und wird bei Ausbleiben dieses Beweises auf Urteilsunfähigkeit erkannt.

\section{Die besondere Stellung der Suizidbeihilfe bei der Beurteilung der Urteilsfähigkeit}

Im Vergleich zu anderen Rechtshandlungen wie z.B. Kauf oder Verkauf eines Bildes stellt das Verlangen nach Suizidbeihilfe den forensischen Psychiater vor besondere Probleme und kann in ihm ein Unbehagen auslösen aufgrund folgender Merkmale:

1. Die Irreversibilität der Entscheidung, auf eine lebensrettende Behandlung zu verzichten, oder der Entscheidung für den Suizid macht einen Fehlentscheid besonders gravierend, denn sie lässt keine nachträgliche Korrektur $\mathrm{zu}$.

2. Der Entscheid zur Lebensbegrenzung aus gesundheitlichen Motiven wird ebenso wie oft auch die Errichtung des Testamentes in der Regel erst im höheren Alter getroffen oder beim Näherrücken des Todes, also im Wissen um eine Diagnose mit hoffnungsloser Prognose. Viele Sterbewillige befinden sich dann in einem Zustand, in dem ihre Fähigkeit vermindert ist, komplizierte Sachverhalte zu erfassen, Beeinflussungsversuche zu erkennen und ihnen zu widerstehen.

3. Eine Überprüfung der Urteilsfähigkeit erfolgt bei Verdacht auf eine Fehlbeurteilung naturgemäss oft erst posthum, und zwar in einem Strafverfahren gegen den Arzt, der Beihilfe geleistet hat. Dabei gilt das Prinzip in dubio pro reo. Daher ist es an der Anklage, einen Zweifel an der Urteilsunfähigkeit auszuschliessen, will sie dem Suizidhelfer eine strafbare Handlung nachweisen.

4. Der psychiatrisch zu beurteilende Sachverhalt, zu dem der Sterbewillige sich zu äussern hat, ist von einer elementaren Schlichtheit. Er zeigt einen geringen Komplexitätsgrad. Der Sterbewillige bekundet gewöhnlich nur den einfachen Willen, sein Leben vorzeitig zu beenden, und er ist dazu auch dann fähig, wenn seine kognitiven Fähigkeiten in einem Ausmass reduziert sind, dass er beispielsweise für die Gründung einer Gesellschaft oder für die Niederschrift eines Testamentes mit vielen möglichen Destinatären überfordert wäre. Beim Aufgeklärtwerden über Krankheitsdiagnose und Prognose müssen auch der vollbesonnene Durchschnittsbürger und manch gebildeter Zeitgenosse oft mehr auf ihr blindes Vertrauen in die Götter in Weiss als auf das Verstehen von deren Erläuterungen, etwa von transplantationschirurgischen und immunmedizinischen Zusammenhängen, abstellen.

5. Die Überlappung der Kriterien für die Einschätzung der Urteilsfähigkeit mit den Voraussetzungskriterien für die Indikation für eine Beihilfe zum Suizid schafft besondere Zuständigkeitsprobleme. Die Merkmale Dauerhaftigkeit, Wohlerwogenheit und Unabhängigkeit von Druckversuchen sind nämlich nicht nur Kriterien für die Zulassung der Beihilfe zum Suizid, sondern bei der Abklärung der Urteilsfähigkeit ebenfalls essentielle Merkmale. Sind sie erfüllt, so kann im Allgemeinen eine Urteilsfähigkeit angenommen werden, wogegen der Umkehrschluss nicht zutrifft. Denn ist der Suizidwillige fähig zu einer dauerhaften, wohlerwogenen und von äusserem Druck unabhängigen Willensbildung, so ist damit noch nicht garantiert, dass er von dieser Fähigkeit Gebrauch macht. So können normalpsychologische, d.h. nicht in krankhaften psychopathologischen Veränderungen wurzelnde konstante Persönlichkeitsmerkmale oder vorübergehende psychische Eigenschaften wie Wankelmütigkeit, Unvorsichtigkeit, Launenhaftigkeit, Erschöpfbarkeit, Impulsivität, Beeinflussbarkeit, Leichtgläubigkeit zu einem Suizidentschluss führen, welcher trotz erhaltener Urteilsfähigkeit im Sinne des Gesetzes die erwähnten Zulassungskriterien (Richtlinien) nicht erfüllt. Dies ist deshalb hervorzuheben, weil im Gegensatz zur Beanspruchung der Beihilfe zum Suizid bei anderen Rechtshandlungen wie einem Kaufvertrag oder der Niederschrift des Testamentes neben der Urteilsfähigkeit keine anderen Kriterien wie Dauerhaftigkeit, Wohlerwogenheit und Unabhängigkeit von äusserem Druck erfüllt werden müssen. Daher ist vor allem aus psychiatrisch-forensischer Sicht auf der zuverlässigen Durchführung der Richtlinienüberprüfung zu bestehen, will man nicht einem zügellosen Suizidbeihilfe-Aktivismus Vorschub leisten.

6. Die im Gesetz vorgegebene alternative Fragestellung «Urteilsfähigkeit ja oder nein?» hat ebenfalls unerwünschte Folgen: Ein nur leichter oder mittlerer Grad der Verminderung der Urteilsfähigkeit führt den psychiatrischen Experten zu einer Postulierung von Urteilsfähigkeit, womit er aus seiner Sicht grünes Licht für die Suizidbeihilfe gibt, die der freiwillig aus dem Leben Scheidende bei völliger Urteilsfähigkeit vielleicht aber nicht erbeten hätte. Die vom Experten gemäss Gesetzesbestimmung grosszügig zugestandene Ur- 
teilsfähigkeit tendiert dazu, die Legitimation der Suizidbeihilfe zu suggerieren, begünstigt durch die sich zunehmend verbreitende unselige Mode, die Zuständigkeit für unangenehme Fragen und Entscheide auf die Psychiatrie abzuwälzen.

7. Auf der anderen Seite verführt die Unexaktheit forensischer Beurteilung der Suizidbeihilfe abgeneigte psychiatrische Experten dazu, ihre eigene politisch und ethisch motivierte Missbilligung der Suizidbeihilfe missbräuchlich durchzusetzen, indem sie Urteilsunfähigkeit in Ausschöpfung ihres erheblichen Ermessensspielraumes postulieren, wo sie gemäss üblicher Anwendung der Kriterien eine Urteilsfähigkeit annehmen müssten.

\section{Psychiatrische Kriterien zur Beurteilung der Urteilsfähigkeit}

Aus einer psychiatrischen Diagnose lässt sich keine Beurteilung der Urteilsfähigkeit ableiten. Es gibt keine psychiatrische Erkrankung, bei der die Urteilsfähigkeit a priori ausgeschlossen wäre, mit Ausnahme von solchen psychischen Störungen, bei denen die Unmöglichkeit eines sinnvollen zwischenmenschlichen Kontaktes Bestandteil der diagnostischen Kriterien ist, wie beispielsweise beim katatonen Stupor oder bei der Idiotie. Von einer Diagnose Schizophrenie, von einer leichten oder mittleren Intelligenzminderung, von einer Drogensucht als Diagnose kann also nicht von vornherein auf eine Urteilsunfähigkeit geschlossen werden. Es bedarf stets einer Einzelfallwürdigung.

Helmchen und Lauber $[13,14]$ arbeiteten folgende vier Kriterien für die erhaltene Urteilsfähigkeit heraus, an denen sich die Beurteilung entscheidet:

1. Fähigkeit, einen bestimmten Sachverhalt zu verstehen.

2. Fähigkeit, die Information in rationaler Weise $\mathrm{zu}$ verarbeiten.

3. Fähigkeit, die Information angemessen zu bewerten.

4. Fähigkeit, den eigenen Willen auf der Grundlage von Verständnis, Verarbeitung und Bewertung der Information zu bestimmen und zu äussern.

\section{Mit Verstehen ist das intellektuelle Erfassen gemeint}

Ein hinreichendes Verstehen ist ausgeschlossen bei:

- örtlicher und situativer Desorientiertheit;

- hochgradiger Merkfähigkeitsstörung (Sekundengedächtnis);

- hochgradiger Gedächtnisstörung;

- ausgeprägter Aufmerksamkeitsstörung;

- Störungen der Bewusstseinshelligkeit in der Aufklärungssituation;
- krankheitsbedingt fehlender oder völlig fehlgehender Wahrnehmung der eigenen Krankheit (hirnorganisch oder psychotisch bedingte Anosognosie).

Hinreichendes Verstehen ist fraglich und genauer zu prüfen bei:

- leichteren zeitlichen Orientierungsstörungen

- ausgeprägter Müdigkeit bis zur Somnolenz

- aphasischen Störungen

- psychotisch (depressiv, manisch, schizophren) bedingten Konzentrations- und Auffassungsstörungen

- Schwachsinn [14].

\section{Rationale Verarbeitung meint die}

Fähigkeit, positive und negative Aspekte von Entscheidungsalternativen gegeneinander abzuwägen [14]

Sie ist ausgeschlossen bei:

- hochgradiger affektiver Vereinseitigung der Beurteilung (Panik, psychotische Angst, psychotisches Misstrauen, depressiver Nihilismus u.ä.);

- wahnhafter Verarbeitung der gegebenen Informationen (bei schizophrenen Erkrankungen, depressivem Wahn, organisch bedingten paranoiden Syndromen).

Sie ist fraglich bei:

- massiver, nicht psychotischer akuter Verleugnung oder Bagatellisierung der eigenen Krankheit;

- Affektlabilität, depressiven, missmutigen, euphorischen, misstrauischen, zornigen Verstimmungen.

Sie ist nicht ausgeschlossen bei:

- überwertigen Ideen (z. B. religiöser, esoterischer, politischer Art);

- Fehlabwägung aus Trotz oder Misstrauen gegen Angehörige oder Behandler.

\section{Die Fähigkeit zu werten meint die} subjektive Wertung im Hinblick auf Wertgefüge und Zukunftsziele

Diese Fähigkeit ist fraglich, wenn:

- der Patient vertraute Personen nicht erkennt oder verkennt (als Indikator fehlender innerer Repräsentanz entscheidender Orientierungspunkte personaler Bewertung);

- der Patient keinen Zugang zu den emotionalen Aspekten der zu treffenden Entscheidung hat (Euphorie, Parathymie, Affektstarre, krankhafte Unernsthaftigkeit);

- der Patient keinen Zugang zu den personalen Implikationen der zu treffenden Entschei- 
dung hat (Unfähigkeit zur Selbstzuordnung, zum Abgleich mit dem Selbstkonzept, mit seiner sozialen Situation);

- der Patient über keine intentionale zeitliche Zuordnungsstruktur verfügt.

Eine Unfähigkeit zu subjektiver Wertung kommt vor, vor allem bei hirnlokalen Psychosyndromen, starker affektiver Wesensänderung, bei geistiger Behinderung/Schwachsinn, bei psychotischen Residualzuständen (schweren Persönlichkeitsstörungen).

\section{Die Willensfähigkeit meint die Fähigkeit zur autonomen Umsetzung von Überlegung in Handlung}

Sie ist eingeschränkt oder aufgehoben z. B. bei Stuporzuständen, hochgradiger Ambivalenz bei Psychose- oder Zwangskranken, Antriebshemmung bei Depressionen, manischen Zuständen mit Flüchtigkeit von Willensbildungen. Sie ist für die Beurteilung von fehlbaren Unterlassungen im Geschäftsleben von Bedeutung und spielt bei der Beihilfe von Suizid eine eher unbedeutende Rolle, da die Unterlassung des Suizides gewöhnlich nicht Gegenstand eines Verfahrens oder einer Beurteilung der Urteilsfähigkeit ist, weil die Unterlassung des Suizids dem Sterbewilligen nicht vorgeworfen wird.

Für die Bitte um Beihilfe zum Suizid ist gemäss Expertenbericht in einer gestraffteren Darstellung der Ausschliessungskriterien eine sog. Einwilligungsunfähigkeit anzunehmen, wenn der Betroffene

- den Wert oder Rang der von seiner Einwilligungsentscheidung berührten Güter und Interessen nicht erfassen kann;

- die sich aus seiner Einwilligungsentscheidung (möglicherweise) ergebenden Folgen oder Risiken nicht abzuschätzen vermag;

- nicht einsehen kann, welche Mittel zur Erreichung seines angestrebten Zieles seine Rechtsgüter oder Interessen weniger belasten würden [1].

\section{Klinisch-psychopathologische Sicht}

Der praktizierende Psychiater wird in der Regel seine Entscheide nicht nach abstrakten und theoretischen Vorgaben richten, sondern seinen gesunden beruflichen Menschenverstand befragen.

Eine Urteilsfähigkeit ist demnach stets zu verneinen, wenn das Motiv zum Suizid aus der geisteskranken Symptomatik stammt. Dies ist der Fall bei imperativen Stimmen, die zu Selbsttötung drängen, bei wahnhaften Zuständen, die den Sterbewunsch hervorrufen, bei depressiven Verstimmungen, die die Todessehnsucht heraufbeschwören, bei neurotischen Persönlichkeiten, die unter dem Einfluss verdrängter Schuldgefühle eine drastische Selbstbestrafung anstreben, bei Krankheiten wie hirnorganischen Veränderungen, Persönlichkeitsstörungen, süchtiger Wesensänderung und Schizophrenia simplex, die eine Gleichgültigkeit und Entdifferenzierung bewirken.

Phasenhafte und episodische Verläufe legen es nahe, jeweils den Habitualzustand abzuwarten, um die Urteilsfähigkeitsbeurteilung vorzunehmen.

Eine Urteilsfähigkeit ist aber andererseits bei psychisch chronisch Kranken möglich, die sich zu ihrer Krankheit in ein bewusstes, reflektierendes Verhältnis zu setzen vermögen und mit ihren gesunden Anteilen ihren Krankheitszustand, ihren Krankheitsverlauf und die sozialen Implikationen besonnen und nachvollziehbar beurteilen.

Eine Standardisierung der Abklärung durch Screeningmethoden und semiquantitative Methoden, die heute oft marktschreierisch angepriesen werden, täuscht eine Qualitätsverbesserung vor und lässt sowohl Experten als auch ihre Auftraggeber sich in fragwürdiger, falscher Sicherheit wiegen. Vor allem die Orientierung des abschliessenden Entscheides nach der Summe der erhobenen Punktwerte kann zu fatalen Fehleinschätzungen führen, indem empathische Verstehensressourcen nicht benutzt und vielsagende anschauliche Eindrücke ignoriert werden.

\section{Abschliessende Bemerkung}

Der psychiatrische Gutachter ermöglicht mit der Bejahung der Urteilsfähigkeit eine Handlung, deren Folgen irreversibel sind, und die, wenn sie unrechtmässig erfolgt ist, zu den moralisch bedenklichsten und im Gesetz am schärfsten geahndeten Delikten gehört. Aus diesem Grunde will er die höchste Sicherheit haben, dass der Verstorbene auch in Zukunft seinen Entschluss und seine Handlung nicht bereuen würde und dass diese auch von seiner Umgebung nicht als voreilig und unvernünftig bewertet werden wird. Die Begutachtung der Urteilsfähigkeit alleine ist noch kein Garant für die Richtigkeit der Entscheidung zur Suizidbeihilfe.

Die Abklärung der Indikation für eine Beihilfe zum Suizid hat aus psychiatrischer Sicht korrekterweise zweigleisig zu erfolgen. Einerseits sind aus medizinischer und psychiatrischer Sicht die Voraussetzungen gemäss den Richtlinien (Wohlerwogenheit, Dauerhaftigkeit, Unheilbarkeit der Krankheit) zu prüfen, wobei auch hier ein psychiatrischer Experte beizuziehen ist, wenn nach dem Verlauf einer psychiatrischen Krankheit gefragt wird. Es ist u. a. zweckmässig, dem Psychia- 
ter nicht nur die Frage nach der Urteilsfähigkeit zu stellen, sondern auch die Frage, ob eine allfällig vorhandene psychiatrische Krankheit den Suizidwillen in Zukunft noch verändern könnte.

Die Frage könnte lauten: Könnten 1. der mögliche Verlauf der psychiatrischen Krankheit/psychischen Störung, 2. zukünftige Veränderungen der Lebenssituation und 3. Möglichkeiten der Behandlung den Suizidwunsch des Patienten in Zukunft ändern? Wenn ja, in welcher Weise?

Andererseits hat unabhängig von dieser Fragestellung eine psychiatrische Begutachtung der Urteilsfähigkeit zu erfolgen, wobei dem Fachexperten die Unterlagen der Abklärungen der Zulassungskriterien wenn möglich zur Verfügung stehen sollen, um unnötige Wiederholungen zu vermeiden.

Bestehen von vornherein schwerwiegende Zweifel an der Urteilsfähigkeit aufgrund einer schweren psychiatrischen Krankheit wie z. B. fortgeschrittene Demenz oder Wahnkrankheit, ist es angebracht, die Urteilsfähigkeit zuerst zu beurteilen. Deren Verneinung würde die gründliche und belastende Überprüfung der Voraussetzungskriterien erübrigen. Dabei ist zu beachten, dass die Überprüfung der Urteilsfähigkeit zum Zeitpunkt des begleiteten Suizides zu wiederholen ist, falls sie längere Zeit zurückliegt.

Die Erfüllung der Sorgfaltspflicht setzt einen Aufwand voraus, der für den Sterbewilligen zu einer wohl unvermeidbaren Belastung wird. Auch dies wird im Diskurs über Suizidbeihilfe nicht einheitlich beurteilt, wie viele andere von mir beleuchtete Aspekte auch. Einigkeit dürfte wohl nur in einer Frage bestehen, nämlich dass es gut wäre, wenn Menschen, die sich suizidiert haben, sanftere Wege hätten wählen können als die oftmals praktizierten brutalen, dilettantischen Methoden.

\section{Literatur}

1 Bosshard G, et al. Expertenbericht z. Hd. von ExitDeutsche Schweiz, Suizidbeihilfe bei Menschen mit psychischen Störungen, unter besonderer Berücksichtigung der Urteilsfähigkeit, vom 18. April 2004.

2 Ernst K. Arzt und Suizid. Schweiz Ärztezeitung. 1994;75:1989-2002.

3 Gmür M. Die Unfähigkeit zu zweifeln. Stuttgart: Klett-Cotta; 2006.

4 Finzen A. Suchtprophylaxe bei psychischen Störungen. Bonn: Thieme; 1989, zitiert nach Fenner D. Sterbehilfe - Grundsätzliche und praktische Fragen. Tagungsdokumentation vom 13. Oktober 2005.

5 Bronisch T. Der Suizid. 4. Auflage. München: C. H. Beck; 1980, zitiert nach Fenner D. Sterbehilfe Grundsätzliche und praktische Fragen. Tagungsdokumentation vom 13. Oktober 2005.

6 Medizinisch-ethische Richtlinien der SAMW. Kapitel 4.1. S. 7.

7 Bundesgerichtsentscheid vom 3. November 2006, 6.34, nach Medizinisch-ethische Richtlinien der SAMW, S. 7.

8 Binder H. Die Urteilsfähigkeit in psychologischer, psychiatrischer und juristischer Sicht. Zürich: Schulthess; 1964.

9 Petermann FT. Urteilsfähigkeit. Zürich: Dike; 2008.

10 Bucher E. Die natürlichen Personen. Berner Kommentar. Band I, 2. Abt., 1. Teilband. Art. 16 N 148 ff. Bern; Stämpfli: 1976.

11 Pedrazzini NM, Oberholzer N. Grundriss des Personenrechts. 4. Auflage. Bern: Stämpfli, 1993. 75 m.N., zitiert nach [1].

12 Brückner C. Das Personenrecht des ZGB. Zürich: Schulthess; 2000. N 164 ff., BGE 124 III 5 mN.; zitiert nach [1].

13 Helmchen H, Lauber H (Hrsg.). Dürfen Ärzte mit Demenzkranken forschen? Stuttgart, New York: Thieme; 1995.

14 Kröber H-L. Psychiatrische Kriterien zur Beurteilung der Einwilligungsfähigkeit. Rechtsmedizin. 1998;8:41-6. 